\title{
The Effect of Telenursing on Referral Rates of Patients With Head Trauma and Their Family's Satisfaction After Discharge
}

\author{
Akram Shahrokhi, MSc $\square$ Jalil Azimian, PhD $\square$ Atousa Amouzegar, MSc $\square$ Sonia Oveisi, PhD
}

\begin{abstract}
We aimed to assess the effect of telenursing on referral rates of patients with head trauma and their family's satisfaction after discharge. Seventy-two patients with head trauma were randomly allocated to equal intervention and control groups. The caregivers in both groups were provided with 1-hr face-to-face training on patients' home care and educational booklets, 2 days before discharge. The cell phone numbers of the telenurse was given to the caregivers of the intervention group. Then, the patients in the intervention group were followed up every week through phone calls by the telenurse for 12 weeks and the patient status checklists were completed. Caregivers in the intervention group could call the telenurse any time they desired. The health status of the control group was followed once by a phone call after 12 weeks. Data were analyzed using SPSS software, version 19.
\end{abstract}

ach year, about 1.7 million head traumas occur in the United States, leading to nearly 52,000 deaths and 275,000 hospitalizations (Hinkle \& Cheever, 2014), about 1.1 million of whom are treated and discharged from hospital (Brunner, Smeltzer, Bare, Hinkle, \& Cheever, 2010), and a considerable number $(80,000-90,000)$ suffer from long-term disability (Hinkle \& Cheever, 2014). During 2012-2013, 55,427 individuals were referred to three main trauma centers in Tehran because of head trauma, who were mainly (74\%) men and the main causes of trauma included road accidents, falls, and beatings (Aligholi \& Safahani, 2015). Head trauma is a major reason of referral to trauma centers in Iran; $18.9 \%$ of admissions in three great teaching hospitals in Tehran, capital of Iran, had occurred because of head

Author Affiliations: Department of Critical Care Nursing (Mss Shahrokhi and Amouzegar), Nursing \& Midwifery Faculty (Dr Azimian), and School of Nursing and Midwifery (Dr Oveisi), Qazvin University of Medical Sciences, Qazvin, Iran.

The authors declare no conflicts of interest.

Correspondence: Atousa Amouzegar, MSc, Critical Care Nursing Department, Nursing \& Midwifery School, Qazvin University of Medical Sciences, Qazvin, Iran (atousa.amouzgar@gmail.com).

DOI: 10.1097/JTN.0000000000000382
Ultimately, 33 patients with a mean $\pm S D$ age of $31.12 \pm$ 10.83 years were studied in the control group and 35 patients with a mean $\pm S D$ age of $34.11 \pm 12.34$ years were studied in the intervention group $(p=.098$ ). The 2 groups differed significantly with respect to referral rates; $39.4 \%$ of the participants in the control group referred to physicians whereas only $25.7 \%$ of the patients in the intervention group needed to refer to physicians ( $p=$ .017). Also, the mean times of referring to a physician differed significantly in both groups. In general, $53.8 \%$ of the caregivers were satisfied with the telenursing program. Follow-up programs using telephone calls were effective and would lead to higher caregiver satisfaction.

\section{Key Words}

Caregivers, Head trauma, Outcome assessment, Patient care, Telenursing

trauma (Ghodsi, Moez Ardalan, \& Daroughehdar, 2002). Incidence rate of head trauma in the central region of Iran was 429 per 100,000 population, so 4,290 patients with head trauma had been admitted to Kashan hospitals during a 2-year period (Fazel et al., 2008).

Head trauma can lead to several complications, ranging from mild concussions to coma and death (Hinkle \& Cheever, 2014). Traumatic brain injury is the leading cause of death and disability worldwide and imposes a great burden on the health care system (Langlois, Rutland-Brown, \& Wald, 2006; Vafaee et al., 2013). The most common complications after head trauma and brain injury include coma, urinary and respiratory tract infections, septicemia, wound injury, meningitis, and brain abscess (Białkowska, Sowa, \& Maksymowicz, 2012; Hinkle \& Cheever, 2014). Many head trauma survivors live with major disabilities, including physical, cognitive, mental, emotional, and behavioral defects (Haffejee, Ntsiea, \& Mudzi, 2013; Vafaee et al., 2013). Considering the complications mentioned, the possibility of readmission increases in patients with traumatic brain injury. Therefore, follow-up is a major part of health care services to establish a consistent and dynamic health care relationship with patients to increase their knowledge and improve the function of patients and their families (Behzad, Bastani, \& Haghani, 2016; 